\title{
ARTICLE
}

Molecular Diagnostics

\section{A review of the most promising biomarkers for early diagnosis and prognosis prediction of tongue squamous cell carcinoma}

\author{
Aisha A. Hussein ${ }^{1}$, Tymour Forouzanfar ${ }^{1}$, Elisabeth Bloemena ${ }^{1}$, JGAM de Visscher $^{1}$, Ruud H. Brakenhoff ${ }^{2}$, C. René Leemans ${ }^{2}$ and \\ Marco N. Helder ${ }^{1}$
}

BACKGROUND: There is a great interest in developing biomarkers to enhance early detection and clinical management of tongue squamous cell carcinoma (TSCC). However, the developmental path towards a clinically valid biomarker remains extremely challenging. Ideally, the initial key step in moving a newly discovered biomarker towards clinical implementation is independent replication. Therefore, the focus of this review is on biomarkers that consistently showed clinical relevance in two or more publications.

METHODS: We searched PubMed database for relevant papers across different TSCC sample sources, i.e., body fluids (saliva, serum/ plasma) and tissues. No restriction regarding the date of publication was applied except for immunohistochemistry (IHC); only studies published between 2010 and June 2017 were included.

RESULTS: The search strategy identified 1429 abstracts, of which 96 papers, examining 150 biomarkers, were eventually included. Of these papers, $66 \%$ were exploratory studies evaluating single or a panel of biomarkers in one publication. Ultimately, based on studies that had undergone validation for their clinical relevance in at least two independent studies, we identified 10 promising candidates, consisting of different types of molecules (IL-6, IL-8, and Prolactin in liquid samples; HIF-1a, SOX2, E-cadherin, vimentin, MALAT1, TP53, and NOTCH1 in tissue biopsies)

CONCLUSIONS: Although more exploratory research is needed with newer methods to identify biomarkers for TSCC, rigorous validation of biomarkers that have already shown unbiased assessment in at least two publications should be considered a high priority. Further research on these promising biomarkers or their combination in multi-institutional studies, could provide new possibilities to develop a specific panel for early diagnosis, prognosis, and individualized treatments.

British Journal of Cancer (2018) 119:724-736; https://doi.org/10.1038/s41416-018-0233-4

\section{BACKGROUND}

Tongue squamous cell carcinoma TSCC is one of the most lethal head and neck cancers worldwide. ${ }^{1}$ It is comparatively silent and progresses from a premalignant state into invasive carcinoma without any specific alarming symptoms. ${ }^{2}$ This causes delay in diagnosis, eventually leading to poor prognosis. The incidence of this disease is rising in the population, particularly in Western communities among young individuals. ${ }^{3-5}$ Unfortunately, even with combined treatment involving surgery, radiation, and chemotherapy, the 5-year survival rate is still unsatisfactory. ${ }^{6,7}$ One reason could be the marked biological propensity for local invasion and the high incidence of cervical lymph node metastasis at initial diagnosis (40\%). ${ }^{8}$ Another is a uniform treatment for all patients with the same clinical and histological features that disregards individual differences in genetic and biological behavior.

Currently, understanding of cancer development and progression is rapidly increasing. Knowledge about specific regulatory pathways and signaling interactions that lead to neoplastic transformation and invasion has been gained. Delineation of these pathways has revealed a multitude of biomolecular changes that could be exploited as biomarkers. A biomarker by definition is an objective measure such as, a gene, a protein, enzyme, or hormone that can reflect the entire spectrum of the disease, from the earliest features to the end stages. It can also provide information on how the body responds to any therapeutic interventions; this may help in making treatment decisions. 9,10

Cancerous cells, or other body cells in response to tumor development secrete or release a subset of biomarkers into tissues and different biological body fluids. The body fluid biomarkers can be detected and evaluated in succession with non-invasive or slightly invasive means, whereas tissues-derived ones need invasive procedures like biopsies. For TSCC, finding a novel, and specific biomarker in body fluids can offer complementary information beyond what is provided by current clinical practice, especially in the field of early detection and diagnosis. Additionally, biomarkers that mirror genetic alterations and proteins expressions on histological slides may play a key role in predicting tongue cancer behavior and determining the treatment plans.

There is a three-level evidence hierarchy for biomarker validation, ranging from exploratory to validated to clinically

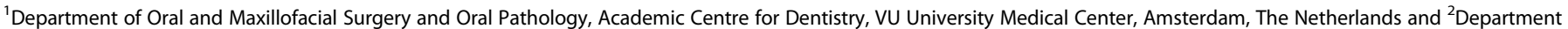
of Otolaryngology-Head and Neck Surgery, VU University Medical Center, Amsterdam, The Netherlands

Correspondence: Marco N. Helder (m.helder@vumc.nl) 
useful, and to qualify as a useful biomarker it is essential to successfully pass them all. The exploratory biomarker is defined as any biomolecule identified in one discovery publication with targeted or untargeted approaches. This classification results in a large list of discovery biomarkers that, however, require rigorous validation. Validation is a second and pivotal step to move any biomarker towards clinical implementation, and is based primarily on confirming a discovery biomarker's finding in at least two independent studies. ${ }^{11,12}$ To date, despite the proposition of a large number of potential biomarkers of TSCC, none are currently used in clinical practice, and only very few have actually proceeded towards the path of validation.

To our knowledge, this review is the first to list the published literature on both liquid and tissue-based biomarkers in TSCC. Since squamous cell carcinoma of different subsites of the oral cavity is quite heterogeneous, we only considered studies that specifically addressed the tongue locus and in particular the mobile part of the tongue. Our focus was particularly on biomarkers whose clinical significance was described in at least two independent studies. As these might represent promising biomarker candidates, we evaluated the studies with regard to the potential of these biomarkers for early diagnosis and prognosis prediction of TSCC, in which the markers demonstrated a consistent association between their expression and specific clinical outcomes. Moreover, we evaluated them using Reporting Recommendations for Tumor Marker Prognostic Studies (REMARK) ${ }^{13}$ guidelines for prognostic studies and STARD ${ }^{14}$ (Standards for Reporting of Diagnostic Accuracy) criteria for the diagnostic ones. In this way, we aim to help both researchers and clinicians in identifying and pursuing the most promising tongue cancer biomarkers for further evaluation and validation studies.

\section{MATERIALS AND METHODS}

Search strategy

Potentially eligible studies were identified in a search of US National Library of Medicine electronic database (PubMed), using combination of the following terms: "tongue carcinoma", "tongue SCC", "biomarker", " biological marker", "tissue", "body fluid " "saliva", "serum/plasma", " immunohistochemistry", "long noncoding (Inc) RNA", and "genetic mutation". No restriction regarding date of publication was applied except for immunohistochemistry (IHC); only studies published between 2010 and June 2017 were included to ensure that all new published evidence on potential markers since the last IHC review ${ }^{15}$ were encompassed. In addition, PubMed Advanced Search Builder (http://www.ncbi. nlm.nih.gov/pubmed/advanced) was utilized to identify some publications. Results were supplemented with manual searching for relevant citations. The initial search was performed in January 2017 and updated in June 2017.

One author (A.A.H.) examined all titles and abstracts to exclude studies that were beyond doubt irrelevant. Then, A.A.H. and M.N.H. assessed full-text manuscripts of all remaining studies against prespecified eligibility criteria.

\section{Selection of studies}

Inclusion criteria.

- Studies investigating association(s) between TSCC and biomarkers

- Studies reporting clinical significance(s) for biomarker expression

- Studies investigating biomarker expressions in oral cavity when all samples were taken from the tongue

- IHC studies encompassing multivariate analysis in statistical assessment

- English full-text version available
Exclusion criteria.

- Studies investigating biomarkers in different anatomical subsites of oral cavity, and head and neck cancer

- Studies unclear about clinical implications

- Studies exclusively addressing the base of the tongue

- Studies investigating biomarkers only in animals

- Studies investigating micro-RNAs as biomarkers; these were already reviewed ${ }^{16}$ recently for their clinical implications in TSCC

- Case reports, letters to the Editor, and systematic reviews

Definition of the level of evidence and promising biomarkers. Biomarkers are usually classified based on the development pipeline, subdivided into 4 phases: exploratory, assay development and validation phase, retrospective validation studies, and prospective validation studies. ${ }^{11,17,18}$ However, since most of the TSCC biomarker studies are still in the exploratory phase with rather small sample sizes, we had to employ an alternative approach, based on the study of Teunissen and co-workers, ${ }^{12}$ which we slightly adapted (downscaled).

Ranking level of evidence (LOE).

- Negative (-): Study reported no significant association between biomarker expression and clinical values

- Weak (+): One study reported an association between biomarker expression and clinical values

- Intermediate $(++): 2$ independent studies reported consistent evidence of an association between biomarker expression and clinical values

- Strong $(+++): \geq 3$ independent studies reported consistent evidence of an association between biomarker expression and clinical values

Only biomarkers with an intermediate or strong $L o E$, i.e., demonstrating a consistent association between their expression and specific clinical outcomes in at least two reports, were considered as promising biomarkers, even in the case that also neutral or opposite predicted outcomes were available for the same biomarker.

Data extraction. Included studies were classified into liquid and tissue-based biomarkers. These were further categorized according to the aforementioned LoE ranking into two groups:

- Group A: studies with negative and weak LoE

- Group B: studies with intermediate and strong LoE

Group B comprised all promising biomarkers, the master variable of interest of the current review. The studies of both groups were arranged according to year of publication, earliest to latest.

Since tissue biopsies were evaluated using various techniques, the tissue-based biomarkers were subdivided as follows:

- Protein biomarkers

- Inc RNA biomarkers

- DNA biomarkers

Information about the biomarker studied, including its usefulness, sample type and size, the method of detection, expression level, type of mutation, and validity indices were listed in table format.

Quality assessment

For the purpose of this review, we first defined a prognostic biomarker as a marker has an association with the typical 
outcomes such as survival rate or recurrence or has an association with the predictor of outcomes like metastasis or tumor grade/size and differentiation. We then started screening the data and found that the vast majority of these studies were prognostic in nature, while a few were diagnostic. Consequently, the quality of the selected biomarkers studies was independently assessed by two authors (A.A.H and M.N.H) on the basis of the criteria as formulated in the Reporting Recommendations for Tumor Marker Prognostic Studies (REMARK) ${ }^{13}$ guidelines for prognostic studies and STARD ${ }^{14}$ (Standards for Reporting of Diagnostic Accuracy) criteria for the diagnostic ones. The former comprises of 20 items, and the latter consists of 30 items, in which each item can encompass several aspects in both guidelines. When all aspects of an item were clearly stated in the study, it was given 1 point, 0.5 point was attributed if some but not all aspects were mentioned, and 0 point were given when the item was not reported. Based on the total scores, the studies were subdivided into three groups: studies with a REMARK score of 15-20 or STARD score of 20-30 were assigned as high reporting quality, studies had a REMARK score of 5-14.5 or STARD score of 10-19.5 were considered to have an average reporting quality, and low reporting quality when the score $\leq 5$ for REMARK and $\leq 10$ for STARD. Disagreements were resolved by discussion.

\section{RESULTS}

A diagram of studies selected for this review after exclusion of irrelevant studies is presented in Fig.(1). Seventy-two studies classified biomarkers belonging to group A, while only 24 studies satisfied the criteria for group B. In total, the included studies examined 150 biomarkers: 23 markers in body fluids, and 127 in tissue. The sample size used in these studies varied between 4 and 202 in group A, and between 17 and 248 in group B. Additionally, quality estimation according to REMARK and STARD (supplementary tables 1 and 2) showed that the overall quality of the included studies was consistent with an average rating.

In thirteen studies, the potential of salivary and blood biomarkers in tongue cancer was evaluated (Table 1). Five of these papers assessed the performance of 14 different markers for early diagnosis, ${ }^{19-23}$ seven assessed performance for prognosis, ${ }^{24-30}$ while the final study, dealing on pro-inflammatory cytokines, assessed both diagnostic and prognostic performance. ${ }^{31}$ Within the included studies, the most promising biomarkers were IL- 6 and IL-8 that showed consistent evidence for clinical usefulness in detection and diagnosis, and prolactin in prognosis. Test accuracy indices were reported in six studies, wherein sensitivity and specificity for these studies ranged from $65 \%-100 \%$ and $45 \%-100 \%$, respectively. In two papers ${ }^{20,22}$ evidence was provided that measuring a single biomarker is less effective than assessing a specific set of biomarkers, the latter showing enhanced sensitivity and specificity.

A total of 83 studies investigated different tissue-biomarkers, using various techniques (Tables 2-4). Forty-nine papers used IHC to assess expression of 82 proteins and their potential usefulness to predict prognosis (Table 2). Fifty-two proteins showed a significant association, and 13 of them were confirmed by mRNA expression. Most IHC studies belonged to group A (39, 80\%). As can be deduced, five markers were independent indicators for good prognosis, while the majority (28) were adverse prognostic indicators. Group B comprised ten studies, identifying four promising IHC biomarkers: HIF-1a, SOX2, E-cadherin, and vimentin.

Using quantitative RT-PCR, eleven studies evaluated IncRNA expression levels in tongue cancerous tissue (Table 3 ). Whereas 16 IncRNAs belonged to group A, only MALAT1 belonged to group B and thus represented the solely promising IncRNA biomarker. Studies assessing DNA mutations in TSCC evaluated 22 mutations in either a single gene or both alleles, while one evaluated promotor methylation of specific genes. Eighteen of these studies
Records identified through database searching ( $n=1429)$

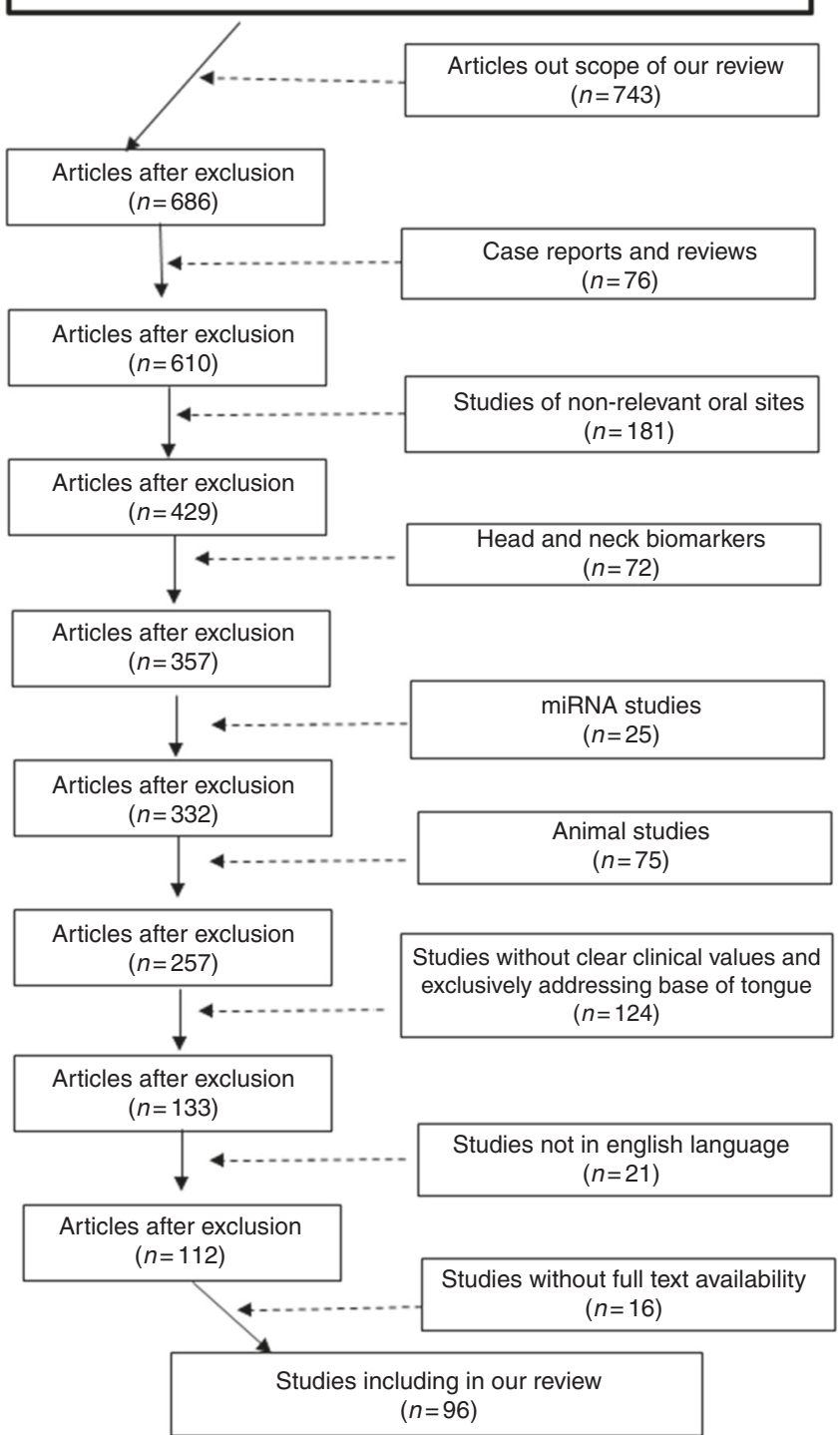

Fig. 1 Flow chart illustrating studies selected

satisfied group A, and five satisfied group B, identifying TP53 and $\mathrm{NOTCH} 1$ as promising mutation markers.

In summary, only 22 biomarkers were evaluated in two or more independent studies, of which only 10 demonstrated a consistent association between their presence and specific clinical outcomes. Of the latter, three were biomarkers for liquid biopsies and seven were tissue-based biomarkers. Collectively, these ten biomarkers qualified as the most promising candidates for tongue cancer diagnosis and prognosis (Fig. 2).

\section{DISCUSSION}

Since pathology and radiology, the current keys to TSCC diagnosis and treatment decisions, are essentially visual subjective measures that are labor-intensive with limitations in diagnostic accuracy, there has been an intensified interest in biomarkers as an objective alternative and more accurate tool for early diagnosis, prognosis, or personalized treatment. A plethora of TSCC biomarker studies have been published, however, virtually all biomarkers are still in early stages of development, and far from potential application in a clinical setting. This review aimed to 


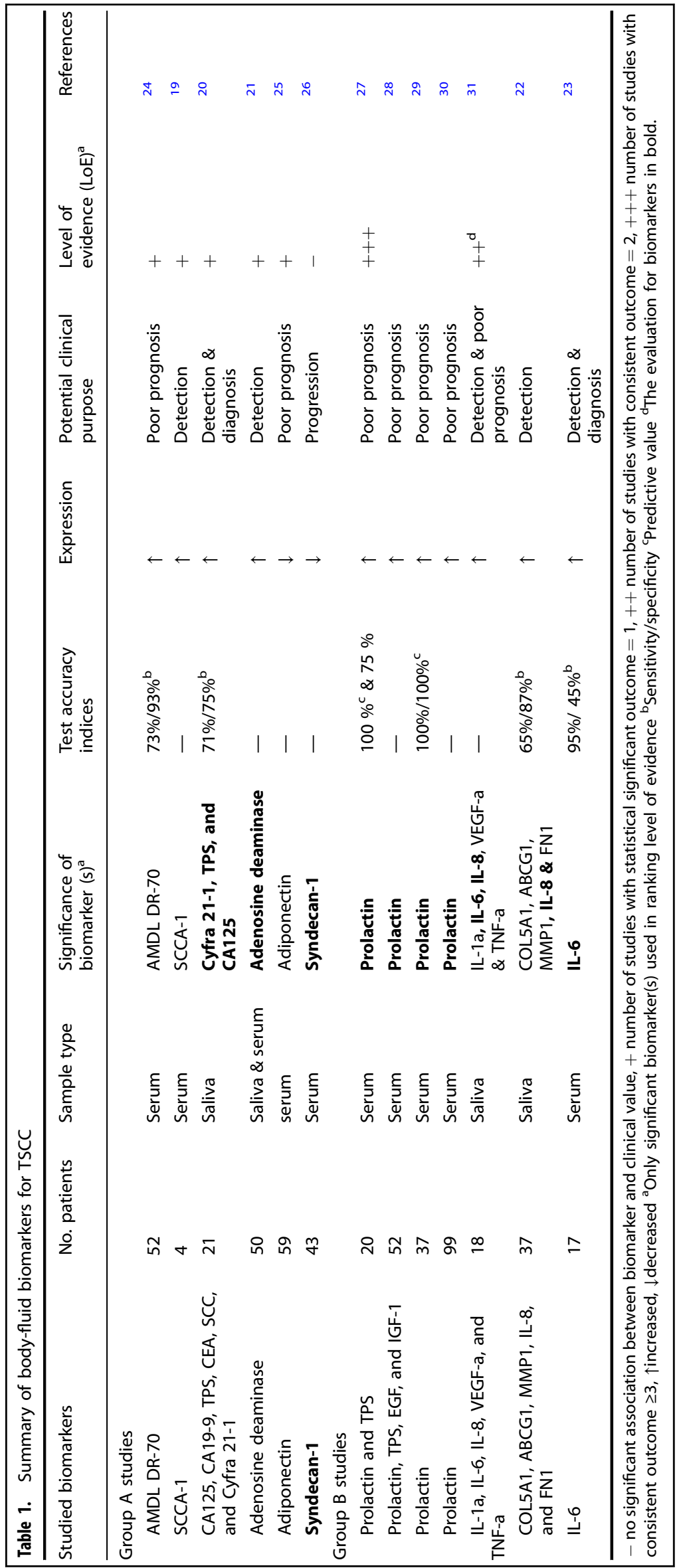


Table 2. Summary of proteins biomarkers for TSCC

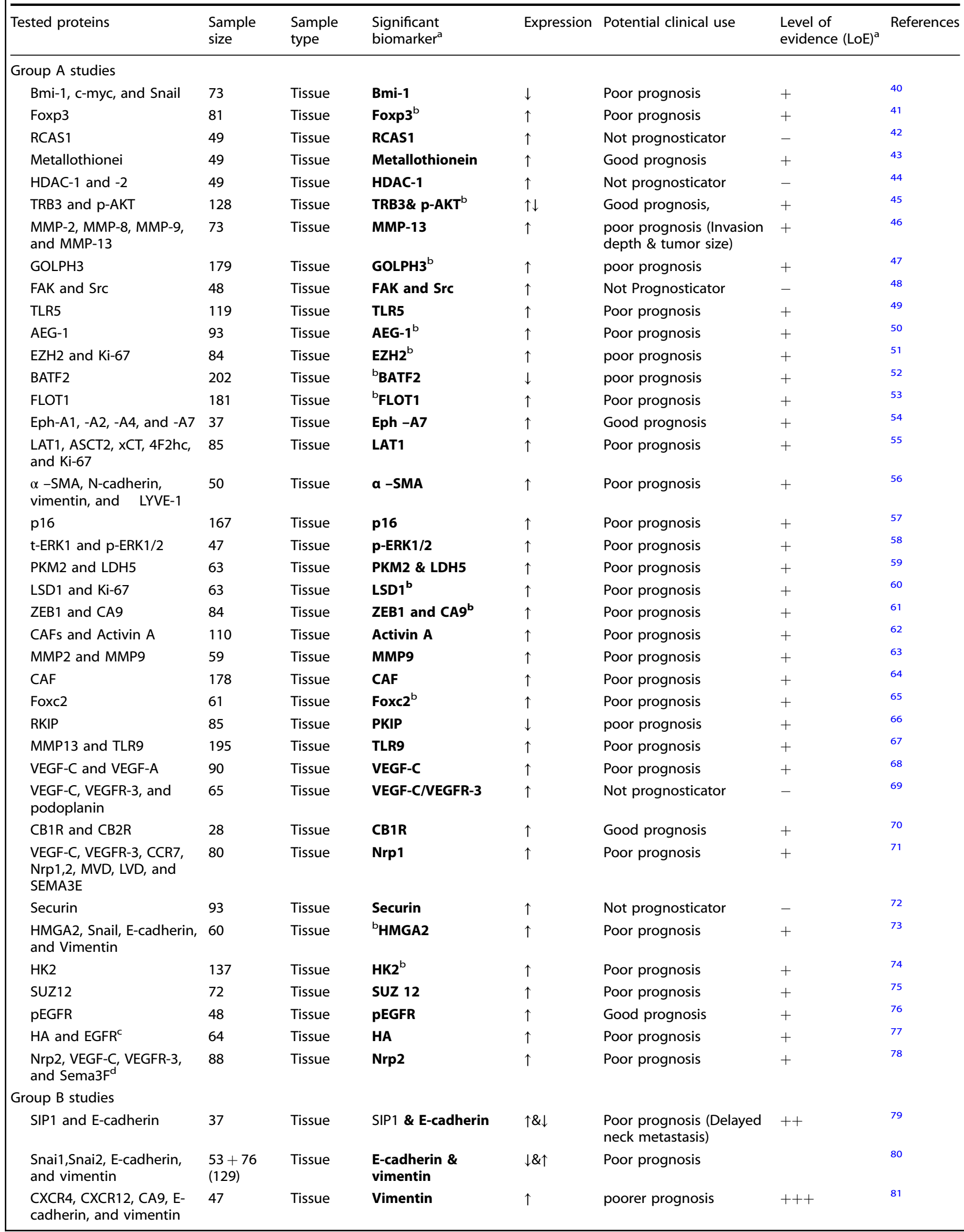




\begin{tabular}{|c|c|c|c|c|c|c|c|}
\hline Tested proteins & $\begin{array}{l}\text { Sample } \\
\text { size }\end{array}$ & $\begin{array}{l}\text { Sample } \\
\text { type }\end{array}$ & $\begin{array}{l}\text { Significant } \\
\text { biomarker }^{\mathrm{a}}\end{array}$ & Expression & Potential clinical use & $\begin{array}{l}\text { Level of } \\
\text { evidence (LoE) })^{a}\end{array}$ & References \\
\hline $\begin{array}{l}\text { HIF-1 } \alpha, \text { HIF-2 } \alpha \text { TWIST2, } \\
\text { and SNIP1 }\end{array}$ & 89 & Tissue & $\begin{array}{l}\text { HIF-1 a, TWIST2 \& } \\
\text { SNIP1 }\end{array}$ & $\uparrow$ & Poor prognosis & +++ & 83 \\
\hline $\begin{array}{l}\text { HIF-1 } \alpha, \text { CA-9, GLUT- } 1 \text {, and } \\
\text { EPOR }\end{array}$ & 33 & Tissue & HIF-1 a & $\uparrow$ & Poor prognosis & & 85 \\
\hline HIF- $1 \alpha$ and VEGF & 49 & Tissue & HIF-1 $a^{b}$ & $\uparrow$ & poor prognosis & & 86 \\
\hline SOX2 & 82 & Tissue & sox2 & $\uparrow$ & Poor Prognosis & ++ & 87 \\
\hline $\begin{array}{l}\text { ALDH1, CD44, OCT4, and } \\
\text { SOX2 }\end{array}$ & 6 & Tissue & SOX2 & $\uparrow$ & Poor prognosis & & 88 \\
\hline
\end{tabular}

drive the acceleration of TSCC biomarker validation by providing an inventory of currently evaluated TSCC biomarkers across different sample sources, including saliva, serum/plasma, and tissues, and by highlighting promising biomarkers that consistently showed clinical relevance in two or more publications.

Overall, we noticed an abundance of studies that described single or multiple biomarkers only in one publication (66\%), whereas there has been no corresponding increase in the validated ones. This may be due to the current pressure from journals to only publish innovative research, which prohibits researchers to perform sound repeat studies providing independent confirmation of the initial identification of a potentially promising biomarker. Since it remains in this exploratory phase pivotal to determine which biomarker is potentially promising and should be prioritized for further steps of confirmation, highquality studies should be performed. In this regard, although we have noticed that the majority of the studied biomarkers in these discovery studies showed significant results, we observed several shortcomings affecting the reliability of their value. For example, in some publications only the data of a small number of patients are presented, while in others study designs are not the optimal or statistical design was unpowered. Two strategies should be implemented to improve this situation: one should emphasize on validation and confirmation of biomarkers that have already shown unbiased assessment in at least one publication, and the other is to conduct future research based on sound scientific and well-planned study designs so that reporting can be done according to guidelines such as REMARK for prognostic biomarkers. ${ }^{13}$

Last year, two other oral cancer biomarker reviews were published (Rivera et al. ${ }^{32}$ Almangush et al. ${ }^{33}$ ). Rivera and coworkers analyzed immunohistochemically identified potential biomarkers for oral SCC at various subsites, thereby however, disregarding the heterogeneity and well-documented variation in genomic and proteomic properties of this malignancy between different regions of the oral cavity, ${ }^{34-36}$ and consequently risking divergence of biomarker specificity and discriminative ability. Also, since their aim was to identify potential biomarkers per se, many biomarkers were evaluated based on one publication. Last but not least, although a scientifically sound method of biomarker evaluation was followed with a quality assessment (QA) according to REMARK guidelines, this QA only indicates the reporting quality of the study, but not necessarily the potential of the biomarker(s) at hand. Almangush et al., on the other hand, evaluated immunohistochemical biomarker studies in TSCC of three decades, and subsequently performed a meta-analysis of the five most frequently studied prognostic biomarkers. Only cyclin D1 and VEGF-A were identified as potential prognostic factors. However, they assessed the overall survival as the clinical end point based on unadjusted or "univariate" analysis, which ignored other known prognostic variables, such as tumor stage, tumor size, etc.

How does our current review relate to the two reviews described above? First of all, in contrast to both other reviews, we evaluated TSCC biomarkers across different sample sources, including saliva, serum/plasma, and tissues. Using this approach, our study identified 10 promising biomarkers, consisting of a different type of molecules: seven proteins, one Inc-RNA, and two genes (Fig. 2). Three of these markers: IL-6, IL-8, and Prolactin were detected in liquid samples, while HIF-1a, SOX2, E-cadherin, vimentin, MALAT1, TP53, and NOTCH1 were identified in tissue biopsies. Secondly, as is also the case for the Almangush review but in contrast to the Rivera report, our focus on a specific subsite within oral cancer, i.e., TSCC, is a clear advanced approach and thus our results may strongly point to unique molecular alterations. These different approaches could also explain why the Rivera paper mentioned 41 potential biomarkers, in which we merely identified ten. Thirdly, Almangush et al. did a comprehensive investigation for published prognostic biomarkers of the last 30 years, while our IHC studies were limited to the published articles in the last 7 years. Due to the technological breakthroughs in the last decade that have enabled scientists to identify new key genes and proteins in tongue carcinogenesis, we deliberately aimed to draw more attention to the latest pursued proteins such as SOX2. Last but not least, we think that a biomarker review should base its evaluation on reports employing multivariate analysis only.

Notably, these 10 promising biomarkers have demonstrated different clinical values. For example, increased expression of serum IL- 6 has been found to effectively discriminate patients with TSCC from controls with an excellent sensitivity. ${ }^{23}$ Likewise, in another study, elevated salivary levels of IL-6 and IL-8 were reported to reliably and accurately identify the progression of TSCC from high-risk to neoplasm. ${ }^{31}$ This implies increased usefulness of combining these two markers in early detection of new or recurrent cases of TSCC. Nevertheless, one should be aware that increased levels of expression may be caused by sources of inflammation elsewhere, and a vigorous effort thus 


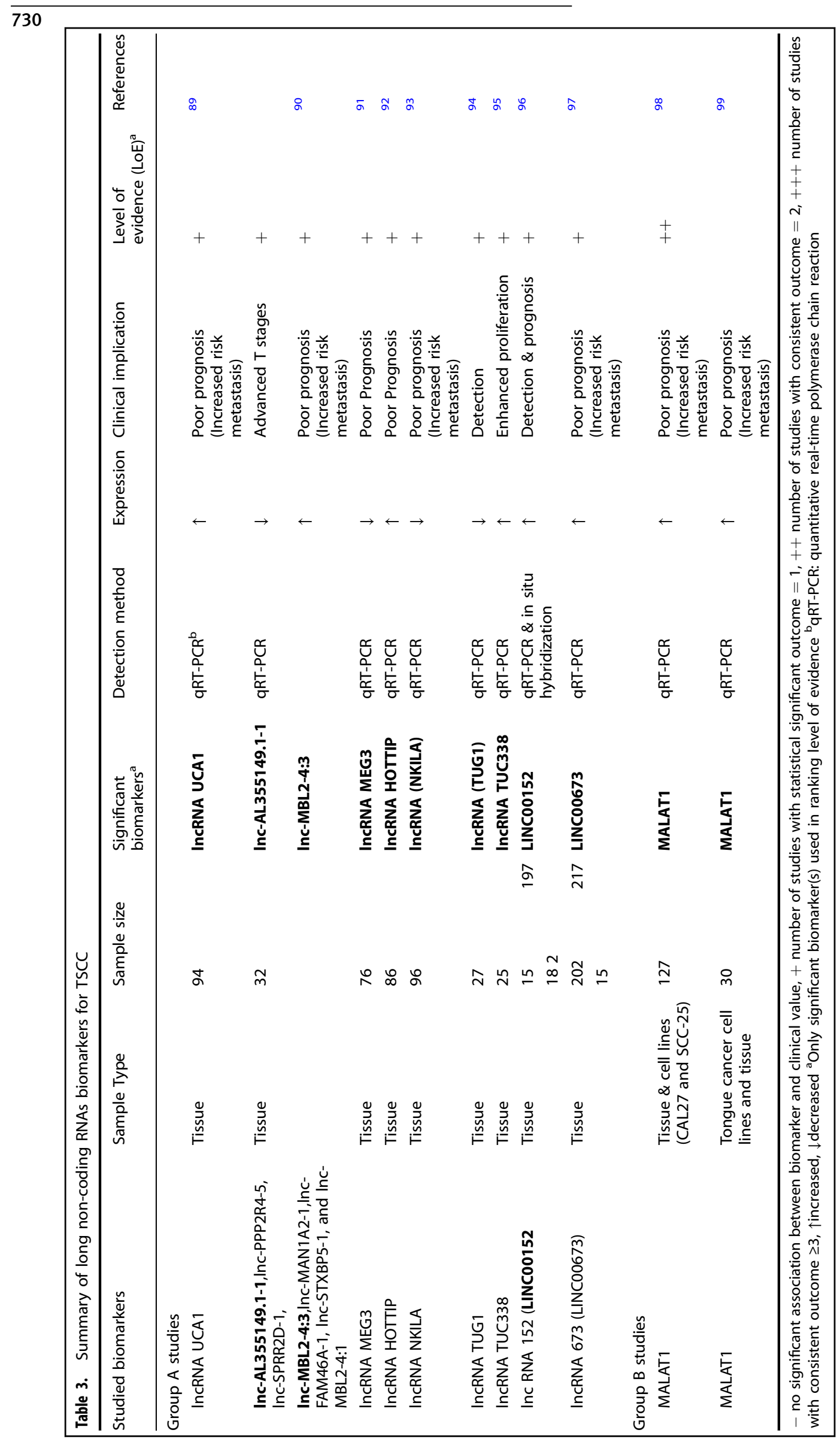




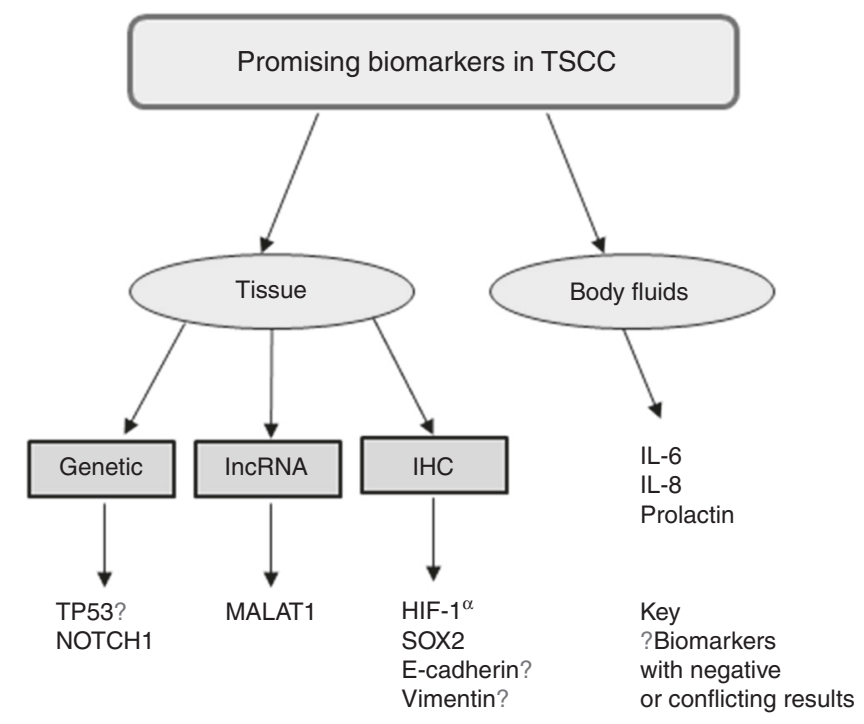

Fig. 2 A diagram illustrating the promising biomarkers

should be made to determine appropriate cutoff values for each marker to differentiate tongue cancer at different stages from healthy subjects. Furthermore, all biomarkers of this list showed a significant correlation with poor prognosis. In clinical practice, applicability of these biomarkers may range from recommending wider surgical resection margins to adjusting management strategy, e.g., the addition of adjuvant chemo-radiation therapy. Another key element to achieve optimal outcome may be through using them as therapeutic targets.

There is no dispute that there is an urgent and yet unmet need for novel diagnostic and prognostic biomarkers to improve TSCC treatment. Therefore, we are convinced that it is timely and highly necessary to integrate all available information about TSCC biomarkers not only from IHC samples but also from other sources. In other words: it could be important to rely on a group of molecules rather than on a single marker, because molecular evidence on multiple levels such genes, proteins, and RNAs may work in concert to prevent or promote the development of the hallmarks of cancer. Only in this sense, it will be possible to form a relatively correct picture about the molecular pathogenesis of this aggressive malignancy and identify which molecules may play a key role and accordingly, may serve as accurate biomarkers. Just as important, limiting the focus to protein expression in IHC studies only could be insufficient and misleading in the biomarker discovery phase, particularly due to the potential ongoing modifications of proteins by a plethora of posttranslation changes. One such example is P53, the most frequent IHC studied protein, which has been reported to have an insignificant value in TSCC prognosis, ${ }^{33}$ whereas we found its gene to be a strong promising indicator. Furthermore, it should be noted that as yet there is no single method suitable for reflecting the complete complexity of TSCC. Hence, our journey through different samples and various molecules assessed by different assays was in our view an essential step to find molecules with distinct biological pathways such as MALAT1 that merit further thorough investigation and validation.

Validation is a critical step for introduction of any newly discovered biomarker into the clinical practice. However, it is important to realize that there are two aspects of validation: clinical and technical. Clinical validation depends on many parameters, one of which is consistency across studies between specific clinical outcomes and the biomarker evaluated, a policy we adopted in our current study. Of equal importance are other clinical parameters that may influence the strength of a biomarker validation. These include the number of cohorts of a study, whether they are of sufficient size or not, existence of a control group, and what their characteristics are. In parallel, technical validation by using independent methods of biomarker evaluation is another parameter that should be strived for.

One major and underappreciated problem with TSCC biomarker studies which we have found is that several studies used very small samples (few with exceptions). Unfortunately, in current practice it is widely accepted that for validation studies the research must meet rigorous criteria in all aspects, particularly in sample size calculation; however, in discovery studies, such criteria are not mandatory. Indeed, neglecting this epidemiological issue in the discovery studies may have contributed to many false findings. And since the discovery studies form an essential element for the selection of biomarkers to be validated, this may partly explain why not one single biomarker has yet reached the oral oncology clinic. Admittedly, including studies with small subjects in this review may potentially bias the conclusions drawn, because the real performance of these biomarkers may remain unclear. However, we consider our validation approach for the promising biomarkers in which two or more cohorts were included as a useful strategy to minimize this bias.

One might argue that our validation approach to focus on the positive consistent studies and ignore the negative ones is considered as flawed and tenuous, particularly if these negative studies may have a higher quality. Therefore, the quality of the included studies was assessed using REMARK and STARD, which are well-established scoring systems to evaluate the quality of prognostic and diagnostic studies, respectively. Nonetheless, it should be mentioned that these two guidelines were primarily developed to assess the quality of reporting rather than to rate the research methodology. According to the evaluation in here, our results showed an average reporting quality for the included studies, which implies that these could be considered trustworthy. As such, we are confident to suggest that our list of promising biomarkers have demonstrated robustness to warrant further validation studies. Notwithstanding, we cannot speculate about the potential for clinical adoption of any of these markers. Further, we noticed that the highest scores were within IncRNA studies. Since all these studies have been published in the recent few years, this might reflect the rise in awareness among researchers about the importance of reporting and transparency in research.

The anterior two-thirds of the tongue (mobile tongue) and the posterior one-third (base of tongue) are commonly considered as two distinct clinical entities, particularly after the recognition of human papillomavirus (HPV) as a risk factor for base of the tongue in $2007 .{ }^{37}$ Indeed, for mobile tongue, no such link with any viruses is found in literature. To date, although each subsite of the tongue is unique with different etiological factors, pathogenesis, and prognosis, unfortunately, many authors still combine the samples of both loci or report their studies without a clear-cut specification. The scarcity of studies prohibited us to strongly apply this distinction, but we would nevertheless strongly recommend specifically addressing the tongue subsites separately.

Intriguingly, tissue-biomarkers could be investigated for its validity for detection of, and screening for TSCC in body fluids. Identification of specific biomolecules in body fluids, with a preference for saliva samples, to obtain on-the-spot potent diagnostic and prognostic information with minimal or noninvasive procedures is still a distant dream. Why this propensity for saliva? Firstly, since saliva is in direct contact with tongue cancer, accumulation of released biomarkers is likely to occur. Secondly, saliva is an ultra-filtrate of plasma, which means that bloodcirculating biomolecules may be detected in saliva as well. Moreover, saliva may be preferred over serum or plasma since the latter may contain biomarker compounds derived from different sources than the actual TSCC. To evaluate the aspects listed above, biomarker levels should preferentially be simultaneously quantified in both saliva and serum/plasma samples. 


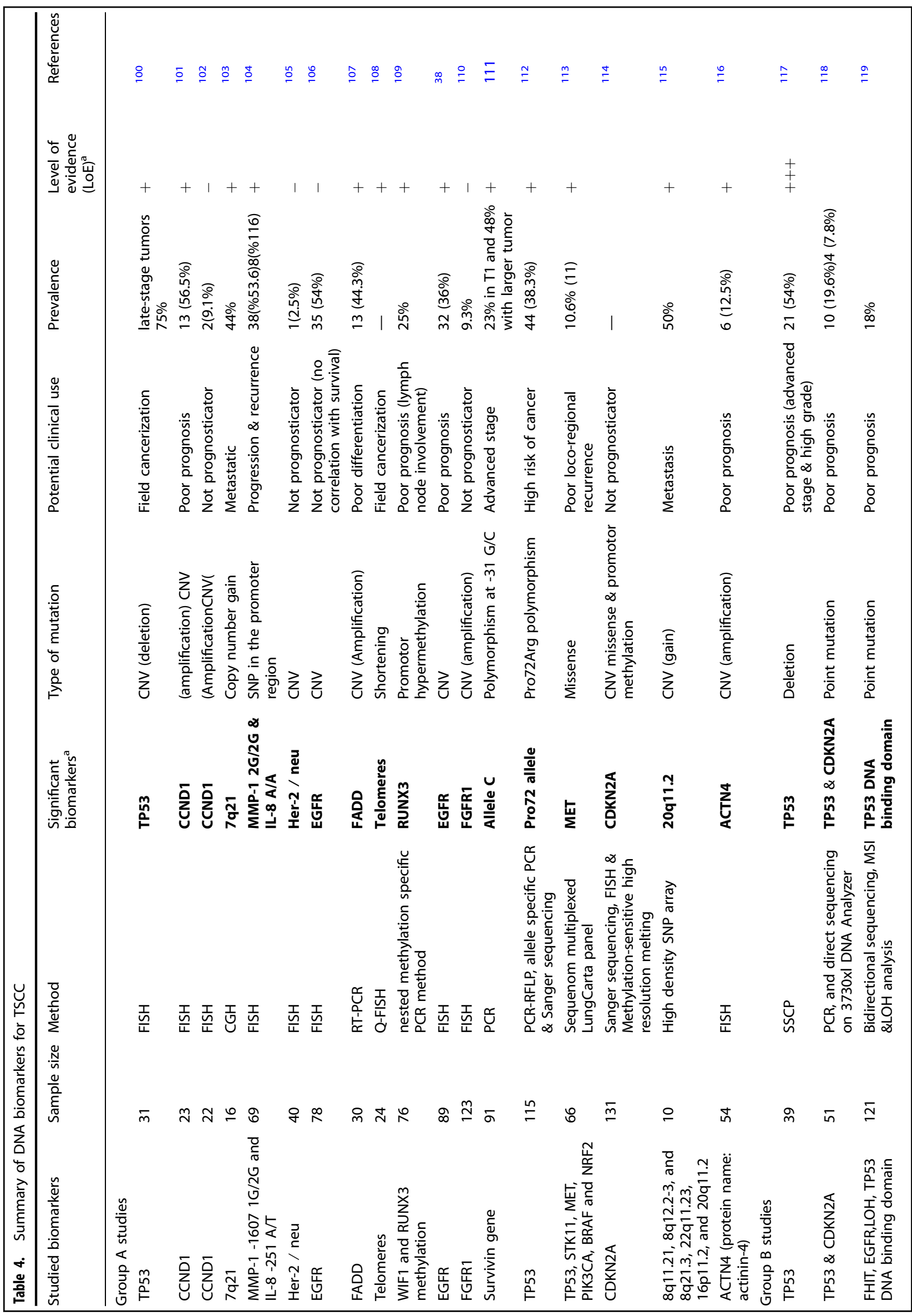


Finally, since biomarkers in body fluids may reflect the entire heterogeneity of cancerous tissue, a biomarker panel instead of a single biomarker may increase sensitivity and specificity. ${ }^{20}$ For example, a single biomarker like pro-inflammatory cytokine IL-6 or IL-8 that holds great promise is often not unique to TSCC, and no reference level of expression has been reached yet in cancer, so combining these markers, together or with other biomarkers, would likely provide a more robust clarification of true detection or prognosis.

Tissue samples are evaluated with various analytical methods, ranging from simple (such as $\mathrm{IHC}$ ) to high technology (such as genomics) platforms. IHC is a relatively simple and affordable technique and consequently, the literature is dominated by this assay type. However, IHC suffers from considerable lack of standardization and mostly only qualitative presentation of data, making technical validation extremely difficult. Nonetheless, developments in digital pathology will improve IHC-based analyses. To solidify our results and compensate for some of these limitations, we only evaluated studies that performed multivariate analysis. Genomic approaches (e.g., microarrays, RT$P C R$ ) are more robust and quantitative methods, with minimum analytical variability and thus facilitating technical validation. Nonetheless, these techniques cannot anticipate levels and actions of the effector molecules (proteins) in directing cancer behavior. $^{38}$ Thus, an integral approach studying genetic mutations, RNA expression, and protein concentrations in parallel may be warranted.

Finally, it is worth mentioning that biomarker development process is financially very challenging, and moving from one phase to another becomes even more burdensome. Recently, it has been estimated that biomarker research expenditures in the U.S only in two years were over $\$ 2.5$ billion, with nearly 500,000 publications. In contrast with this significant and massive investment in biomarker research, the number of translatable biomarkers to patients care is so far negligible. ${ }^{39}$ Regarding tongue cancer biomarkers, we did not find information about (industrial) financial investment, but the pattern appears similar: an overwhelming number of literature studies of potential TSCC biomarkers with no biomarker translation yet to be expected. In this view, we recommend focusing efforts on a selected set of promising biomolecules already in an early phase in order to move clinical biomarker implementation forward in an economically viable manner.

To the best of our knowledge, this is the first and largest review that evaluated specifically TSCC biomarkers across different sources, including saliva, serum/plasma, and tissues in an integral manner. The included studies used various types of assays for analysis, which allowed us to explore more details about the currently evaluated TSCC biomarkers. In addition, based on a staged approach of a biomarker validation in which one publication does not provide a meaningful role of the biomarkers as a measure of disease activity, unless more consistent evidence is available supporting its utility, we used the wide and comprehensive set of data identified here provided a shortlist of qualifying promising biomarkers. Nevertheless, our findings should be understood in the context of some limitations, which may have introduced some bias in our assessments. Firstly, we did not consider the number of patients tested in our evidence rating of the promising biomarkers due to the scarcity of the subjects in several studies. Secondly, we have included IHC studies only from 2010 onwards, consequently, it cannot be excluded that some confirmatory studies for some protein biomarkers were missed. Another limitation is that our search strategy is based on the PUBMED search engine only, which may not have revealed all relevant studies. Furthermore, validation of a biomarker such as a prolactin that emerged as one of the promising biomarkers in this review was entirely based on several studies from the same authors and this reduces the robustness of the finding. Even 
though, the authors followed the rule of thumb by increasing number of the patients in the confirmatory studies, further elucidation in different patient cohorts performed by different research groups to evaluate its value in forecasting prognosis should be conducted.

In conclusion, although biomarkers may play an important role in TSCC detection and management, the developmental path towards a clinically valid biomarker is always long and challenging. This study sheds some very critical light on TSCC biomarkers that demonstrated a consistent association between their expression and specific clinical outcomes at least in two publication, thus qualifying as promising candidates. Furthermore, the findings from this work show how important is the performance of the biomarker during the discovery stage because it will guide the selection of the promising markers for validation. Henceforth, it is critical at this stage to use appropriate sample size and study design. Unfortunately, two-thirds of TSCC biomarker studies have not yet advanced beyond the discovery phase. Despite the fact that more exploratory research is needed to identify specific biomarkers for TSCC, rigorous validation of biomarkers that have already shown unbiased assessment in two publications should be considered a high priority. Further research on these promising biomarkers or their combination in multi-institutional studies, could provide new possibilities to develop a specific panel that may yield better assessment of progression of this malignancy at various stages.

\section{AUTHOR CONTRIBUTIONS}

Conception and design: T.F., J.D.V., and M.N.H.; data acquisition: A.A.H.; quality assessment: A.A.H. and M.N.H.; analysis and interpretation of the data: A.A.H. and M.N. H.; manuscript preparation: A.A.H. and M.N.H.; manuscript editing: E.B., T.F., and J.D.V.; and manuscript reviewing: R.H.B., C.R.L., and E.B.

\section{ADDITIONAL INFORMATION}

Supplementary information is available for this paper at https://doi.org/10.1038/ s41416-018-0233-4.

Competing interests: The authors declare no competing interests.

Funding: No funding other than institutional funding was received

Note: This work is published under the standard license to publish agreement. After 12 months the work will become freely available and the license terms will switch to a Creative Commons Attribution 4.0 International (CC BY 4.0).

\section{REFERENCES}

1. Vigneswaran, N. \& Williams, M. D. Epidemiologic trends in head and neck cancer and aids in diagnosis. Oral. Maxillofac. Surg. Clin. North. Am. 26, 123-141 (2014).

2. Mithani, S. K., Mydlarz, W. K., Grumbine, F. L., Smith, I. M. \& Califano, J. A. Molecular genetics of premalignant oral lesions. Oral. Dis. 13, 126-133 (2007).

3. Tota, J. E. et al. Rising incidence of oral tongue cancer among white men and women in the United States, 1973-2012. Oral. Oncol. 67, 146-152 (2017).

4. Moore, S. R., Johnson, N. W., Pierce, A. M. \& Wilson, D. F. The epidemiology of tongue cancer: a review of global incidence. Oral. Dis. 6, 75-84 (2000).

5. Hussein, A. A. et al. Global incidence of oral and oropharynx cancer in patients younger than 45 years versus older patients: a systematic review. Eur. J. Cancer 82, 115-127 (2017).

6. Brenner, $\mathrm{H}$. Long-term survival rates of cancer patients achieved by the end of the 20th century: a period analysis. Lancet 360, 1131-1135 (2002).

7. Shiboski, C. H., Schmidt, B. L. \& Jordan, R. C. Tongue and tonsil carcinoma: increasing trends in the U.S. population ages 20-44 years. Cancer 103, 1843-1849 (2005).

8. O-charoenra, P. et al. Tumour thickness predicts cervical nodal metastases and survival in early oral tongue cancer. Oral Oncol. 39, 386-390 (2003).

9. Klausner R. D. The Nation's Investment in Cancer Research: A Plan and Budget Proposal for fiscal year 2003. The National Cancer Institute, Bethesda, MD, 2002. https://www.cancer.gov/about-nci/budget/about-annual-plan/nci-plan-2002.pdf
10. Bhatt, A. N., Mathur, R., Farooque, A., Verma, A. \& Dwarakanath, B. S. Cancer biomarkers-current perspectives. Indian J. Med. Res. 132, 129-149 (2010).

11. Comabella, M. \& Montalban, X. Body fluid biomarkers in multiple sclerosis. Lancet Neurol. 13, 113-126 (2014).

12. Teunissen, C. E., Malekzadeh, A., Leurs, C., Bridel, C. \& Killestein, J. Body fluid biomarkers for multiple sclerosis-the long road to clinical application. Nat. Rev. Neurol. 11, 585-596 (2015).

13. McShane, L. M. et al. REporting recommendations for tumour MARKer prognostic studies (REMARK). Br. J. Cancer 93, 387-391 (2005).

14. Bossuyt, P. M. et al. STARD 2015: an updated list of essential items for reporting diagnostic accuracy studies. Clin. Chem. 61, 1446-1452 (2015).

15. Oliveira, L. R. \& Ribeiro-Silva, A. Prognostic significance of immunohistochemical biomarkers in oral squamous cell carcinoma. Int. J. Oral. Maxillofac. Surg. 40, 298-307 (2011).

16. $\mathrm{Yu}, \mathrm{X}$. \& $\mathrm{Li}, \mathrm{Z}$. MicroRNA expression and its implications for diagnosis and therapy of tongue squamous cell carcinoma. J. Cell. Mol. Med. 20, 10-16 (2016).

17. Mordente, A., Meucci, E., Martorana, G. E. \& Silvestrini, A. Cancer biomarkers discovery and validation: state of the art, problems and future perspectives. Adv. Exp. Med. Biol. 867, 9-26 (2015).

18. Pavlou, M. P., Diamandis, E. P. \& Blasutig, I. M. The long journey of cancer biomarkers from the bench to the clinic. Clin. Chem. 59, 147-157 (2013).

19. Huang, $X$. et al. Serum proteomics study of the squamous cell carcinoma antigen 1 in tongue cancer. Oral. Oncol. 42, 26-31 (2006).

20. Nagler, R., Bahar, G., Shpitzer, T. \& Feinmesser, R. Concomitant analysis of salivary tumor markers--a new diagnostic tool for oral cancer. Clin. Cancer Res. 12, 3979-3984 (2006).

21. Rai, B., Kaur, J., Jacobs, R. \& Anand, S. C. Adenosine deaminase in saliva as a diagnostic marker of squamous cell carcinoma of tongue. Clin. Oral. Investig. 15, 347-349 (2011).

22. Suresh, A. et al. Resistance/response molecular signature for oral tongue squamous cell carcinoma. Dis. Markers 32, 51-64 (2012).

23. Lotfi, A. et al. Serum level of interleukin- 6 in patients with oral tongue squamous cell carcinoma. Iran. J. Otorhinolaryngol. 27, 207-211 (2015).

24. Li, X, Qiao, Z, Long, X, Wei, J. \& Cheng, Y. Serum concentration of AMDL DR-70 for the diagnosis and prognosis of carcinoma of the tongue. Br. J. Oral. Maxillofac. Surg. 43, 513-515 (2005).

25. Guo, X. H. et al. Decreased adiponectin level is associated with aggressive phenotype of tongue squamous cell carcinoma. Cancer Sci. 104, 206-213 (2013).

26. Shegefti, M. S. et al. Reduced serum levels of syndecan-1 in patients with tongue squamous cell carcinoma. Laryngoscope 126, E191-E195 (2016).

27. Bhatavdekar, J. M., Patel, D. D., Vora, H. H. \& Balar, D. B. Circulating prolactin and TPS in monitoring the clinical course of male patients with metastatic tongue cancer: a preliminary study. Anticancer Res. 13, 237-240 (1993).

28. Bhatavdekar, J. M., Patel, D. D., Vora, H. H. \& Balar, D. B. Circulating markers and growth factors as prognosticators in men with advanced tongue cancer. Tumour Biol. 14, 55-58 (1993)

29. Bhatavdekar, J. M. et al. Prolactin: its role in advanced tongue cancer. J. Surg. Oncol. 57, 115-120 (1994).

30. Bhatavdekar, J. M. et al. Prolactin as a local growth promoter in patients with locally advanced tongue cancer: GCRI experience. Head. Neck 22, 257-264 (2000).

31. Korostoff, A., Reder, L., Masood, R. \& Sinha, U. K. The role of salivary cytokine biomarkers in tongue cancer invasion and mortality. Oral. Oncol. 47, 282-287 (2011).

32. Rivera, C., Oliveira, A. K., Costa, R. A. P., De Rossi, T. \& Paes Leme, A. F. Prognostic biomarkers in oral squamous cell carcinoma: a systematic review. Oral. Oncol. 72, 38-47 (2017)

33. Almangush, A. et al. Prognostic biomarkers for oral tongue squamous cell carcinoma: a systematic review and meta-analysis. Br. J. Cancer 117, 856-866 (2017).

34. Sathyan, K. M. et al. Carcinoma of tongue and the buccal mucosa represent different biological subentities of the oral carcinoma. J. Cancer Res. Clin. Oncol. 132, 601-609 (2006)

35. Trivedi, T. I. et al. Identification of site-specific prognostic biomarkers in patients with oral squamous cell carcinoma. Neoplasma 58, 217-226 (2011).

36. Mahdey, H. M. et al. Cyclin D1 amplification in tongue and cheek squamous cell carcinoma. Asian Pac. J. Cancer Prev. 12, 2199-2204 (2011).

37. Ramqvist, T., Grun, N. \& Dalianis, T. Human papillomavirus and tonsillar and base of tongue cancer. Viruses 7, 1332-1343 (2015).

38. Nakata, Y. et al. EGFR gene copy number alteration is a better prognostic indicator than protein overexpression in oral tongue squamous cell carcinomas. Eur. J. Cancer 47, 2364-2372 (2011) 
39. Ptolemy, A. S. \& Rifai, N. What is a biomarker? Research investments and lack of clinical integration necessitate a review of biomarker terminology and validation schema. Scand. J. Clin. Lab. Invest. Suppl. 242, 6-14 (2010).

40. Hayry, V. et al. Bmi-1 expression predicts prognosis in squamous cell carcinoma of the tongue. Br. J. Cancer 102, 892-897 (2010).

41. Liang, Y. J. et al. Foxp3 expressed by tongue squamous cell carcinoma cells correlates with clinicopathologic features and overall survival in tongue squamous cell carcinoma patients. Oral. Oncol. 47, 566-570 (2011).

42. Theocharis, S. et al. RCAS1 expression in mobile tongue squamous cell carcinoma: an immunohistochemical study. Med. Sci. Monit. 17, BR228-BR234 (2011).

43. Theocharis, S. et al. Metallothionein expression in mobile tongue squamous cell carcinoma: associations with clinicopathological parameters and patient survival. Histopathology 59, 514-525 (2011).

44. Theocharis, S. et al. Histone deacetylase- 1 and -2 expression in mobile tongue squamous cell carcinoma: associations with clinicopathological parameters and patients survival. J. Oral. Pathol. Med. 40, 706-714 (2011).

45. Zhang, J. et al. TRB3 overexpression due to endoplasmic reticulum stress inhibits AKT kinase activation of tongue squamous cell carcinoma. Oral. Oncol. 47, 934-939 (2011).

46. Makinen, L. K. et al. Prognostic significance of matrix metalloproteinase-2, $-8,-9$, and -13 in oral tongue cancer. J. Oral. Pathol. Med. 41, 394-399 (2012).

47. $\mathrm{Li}, \mathrm{H}$. et al. GOLPH3 overexpression correlates with tumor progression and poor prognosis in patients with clinically NO oral tongue cancer. J. Transl. Med. 10, 168 (2012).

48. Theocharis, S. et al. FAK and Src expression in mobile tongue squamous cell carcinoma: associations with clinicopathological parameters and patients survival. J. Cancer Res. Clin. Oncol. 138, 1369-1377 (2012).

49. Kauppila, J. H., Mattila, A. E., Karttunen, T. J. \& Salo, T. Toll-like receptor 5 (TLR5) expression is a novel predictive marker for recurrence and survival in squamous cell carcinoma of the tongue. Br. J. Cancer 108, 638-643 (2013).

50. Ke, Z. F. et al. Expression characteristics of astrocyte elevated gene-1 (AEG-1) in tongue carcinoma and its correlation with poor prognosis. Cancer Epidemiol. 37, 179-185 (2013).

51. $\mathrm{Li}, \mathrm{Z}$. et al. The polycomb group protein $\mathrm{EZH} 2$ is a novel therapeutic target in tongue cancer. Oncotarget 4, 2532-2549 (2013).

52. Wen, $\mathrm{H}$. et al. Decreased expression of BATF2 is significantly associated with poor prognosis in oral tongue squamous cell carcinoma. Oncol. Rep. 31, 169-174 (2014)

53. $\mathrm{Li}, \mathrm{H}$. et al. Prognostic significance of Flotillin 1 expression in clinically $\mathrm{N} 0$ tongue squamous cell cancer. Int. J. Clin. Exp. Pathol. 7, 996-1003 (2014).

54. Theocharis, S. et al. Ephrin receptor (Eph) -A1, -A2, -A4 and -A7 expression in mobile tongue squamous cell carcinoma: associations with clinicopathological parameters and patients survival. Pathol. Oncol. Res. 20, 277-284 (2014).

55. Toyoda, M. et al. Prognostic significance of amino-acid transporter expression (LAT1, ASCT2, and xCT) in surgically resected tongue cancer. Br. J. Cancer 110, 2506-2513 (2014).

56. Ding, L. et al. alpha-smooth muscle actin-positive myofibroblasts, in association with epithelial-mesenchymal transition and lymphogenesis, is a critical prognostic parameter in patients with oral tongue squamous cell carcinoma. J. Oral. Pathol. Med. 43, 335-343 (2014).

57. Ramshankar, V., Soundara, V. T., Shyamsundar, V., Ramani, P. \& Krishnamurthy, A. Risk stratification of early stage oral tongue cancers based on HPV status and p16 immunoexpression. Asian Pac. J. Cancer Prev. 15, 8351-8359 (2014).

58. Theocharis, S. et al. Extracellular signal-regulated kinase (ERK) expression and activation in mobile tongue squamous cell carcinoma: associations with clinicopathological parameters and patients survival. Tumour Biol. 35, 6455-6465 (2014).

59. Yuan, C. et al. Overexpression of metabolic markers PKM2 and LDH5 correlates with aggressive clinicopathological features and adverse patient prognosis in tongue cancer. Histopathology 65, 595-605 (2014).

60. Yuan, C. et al. High expression of the histone demethylase LSD1 associates with cancer cell proliferation and unfavorable prognosis in tongue cancer. J. Oral. Pathol. Med. 44, 159-165 (2015).

61. Zheng, G. et al. ZEB1 transcriptionally regulated carbonic anhydrase 9 mediates the chemoresistance of tongue cancer via maintaining intracellular $\mathrm{pH}$. Mol. Cancer 14, 84 (2015).

62. Kelner, $N$. et al. Activin $A$ immunoexpression as predictor of occult lymph node metastasis and overall survival in oral tongue squamous cell carcinoma. Head. Neck 37, 479-486 (2015).

63. Aparna, M., Rao, L., Kunhikatta, V. \& Radhakrishnan, R. The role of MMP-2 and MMP-9 as prognostic markers in the early stages of tongue squamous cell carcinoma. J. Oral. Pathol. Med. 44, 345-352 (2015).

64. $\mathrm{Li}, \mathrm{H}$. et al. Cancer-associated fibroblasts provide a suitable microenvironment for tumor development and progression in oral tongue squamous cancer. J. Transl. Med. 13, 198 (2015).
65. Imayama, N. et al. FOXC2 expression is associated with tumor proliferation and invasion potential in oral tongue squamous cell carcinoma. Pathol. Oncol. Res. 21, 783-791 (2015).

66. Hu, F. et al. Clinicopathological features and prognostic implications of Raf kinase inhibitor protein downregulation in tongue squamous cell carcinoma. Oncol. Lett. 10, 1303-1308 (2015).

67. Kauppila, J. H. et al. Toll-like receptor 9 mediates invasion and predicts prognosis in squamous cell carcinoma of the mobile tongue. J. Oral. Pathol. Med. 44 571-577 (2015).

68. Matsui, T., Shigeta, T., Umeda, M., \& Komori, T. Vascular endothelial growth factor C (VEGF-C) expression predicts metastasis in tongue cancer. Oral Surg. Oral Med. Oral Pathol. Oral Radiol. 120, 436-442 (2015).

69. Naruse, T. et al. Immunohistochemical study of vascular endothelial growth factor-C/vascular endothelial growth factor receptor-3 expression in oral tongue squamous cell carcinoma: correlation with the induction of lymphangiogenesis. Oncol. Lett. 10, 2027-2034 (2015).

70. Theocharis, S. et al. Evaluation of cannabinoid $C B 1$ and $C B 2$ receptors expression in mobile tongue squamous cell carcinoma: associations with clinicopathological parameters and patients' survival. Tumour Biol. 37, 3647-3656 (2016).

71. Al-Shareef, H. et al. Use of NRP1, a novel biomarker, along with VEGF-C, VEGFR-3, CCR7 and SEMA3E, to predict lymph node metastasis in squamous cell carcinoma of the tongue. Oncol. Rep. 36, 2444-2454 (2016).

72. Heikkinen, l. et al. Does securin expression have significance in prognostication of oral tongue cancer? A pilot study. Eur. Arch. Otorhinolaryngol. 273, 3905-3911 (2016).

73. Zhao, X. P. et al. Overexpression of HMGA2 promotes tongue cancer metastasis through EMT pathway. J. Transl. Med. 14, 26 (2016).

74. Wang, W. et al. Hexokinase 2 enhances the metastatic potential of tongue squamous cell carcinoma via the $\mathrm{SOD} 2-\mathrm{H} 2 \mathrm{O} 2$ pathway. Oncotarget 8 , 3344-3354 (2017)

75. Hu, H. et al. Overexpression of suppressor of zest 12 is associated with cervical node metastasis and unfavorable prognosis in tongue squamous cell carcinoma. Cancer Cell. Int. 17, 26 (2017).

76. Theocharis, S. et al. Phosphorylated epidermal growth factor receptor expression is associated with clinicopathologic parameters and patient survival in mobile tongue squamous cell carcinoma. J. Oral. Maxillofac. Surg. 75, 632-640 (2017).

77. Lindell Jonsson, E., Nylander, K., Hallen, L. \& Laurell, G. Immunohistochemical analysis of EGFR and hyaluronan in tongue cancer and the development of regional recurrence in patients initially diagnosed NO. Acta Otolaryngol. 137, 877-882 (2017).

78. Ong, H. S. et al. Cytoplasmic neuropilin 2 is associated with metastasis and a poor prognosis in early tongue cancer patients. Int. J. Oral. Maxillofac. Surg. 46, 1205-1219 (2017)

79. Sakamoto, K. et al. Overexpression of SIP1 and downregulation of E-cadherin predict delayed neck metastasis in stage $\mathrm{I} / \mathrm{II}$ oral tongue squamous cell carcinoma after partial glossectomy. Ann. Surg. Oncol. 19, 612-619 (2012).

80. Wang, C. et al. Deregulation of Snai2 is associated with metastasis and poor prognosis in tongue squamous cell carcinoma. Int. J. Cancer 130, 2249-2258 (2012).

81. Albert, S. et al. Prognostic value of the chemokine receptor CXCR4 and epithelial-to-mesenchymal transition in patients with squamous cell carcinoma of the mobile tongue. Oral. Oncol. 48, 1263-1271 (2012).

82. Liu, P. F. et al. Vimentin is a potential prognostic factor for tongue squamous cell carcinoma among five epithelial-mesenchymal transition-related proteins. PLoS ONE 12, e0178581 (2017).

83. Liang, X. et al. Hypoxia-inducible factor-1 alpha, in association with TWIST2 and SNIP1, is a critical prognostic factor in patients with tongue squamous cell carcinoma. Oral. Oncol. 47, 92-97 (2011).

84. Huang, C. et al. Association of increased ligand cyclophilin A and receptor CD147 with hypoxia, angiogenesis, metastasis and prognosis of tongue squamous cell carcinoma. Histopathology 60, 793-803 (2012).

85. Han, M. W. et al. Role of FDG-PET as a biological marker for predicting the hypoxic status of tongue cancer. Head. Neck 34, 1395-1402 (2012).

86. Kang, F. W., Gao, Y., Que, L., Sun, J. \& Wang, Z. L. Hypoxia-inducible factor-1alpha overexpression indicates poor clinical outcomes in tongue squamous cell carcinoma. Exp. Ther. Med. 5, 112-118 (2013).

87. Du, L. et al. Sox2 nuclear expression is closely associated with poor prognosis in patients with histologically node-negative oral tongue squamous cell carcinoma. Oral. Oncol. 47, 709-713 (2011).

88. Huang, C. F., Xu, X. R., Wu, T. F., Sun, Z. J. \& Zhang, W. F. Correlation of ALDH1, CD44, OCT4 and SOX2 in tongue squamous cell carcinoma and their association with disease progression and prognosis. J. Oral. Pathol. Med. 43, 492-498 (2014). 
89. Fang, Z. et al. Increased expression of the long non-coding RNA UCA1 in tongue squamous cell carcinomas: a possible correlation with cancer metastasis. Oral Surg. Oral Med. Oral Pathol. Oral Radiol. 117, 89-95 (2014).

90. Gao, W., Chan, J. Y. \& Wong, T. S. Long non-coding RNA deregulation in tongue squamous cell carcinoma. Biomed. Res. Int. 2014, 405860 (2014).

91. Jia, L. F. et al. Expression, regulation and roles of miR-26a and MEG3 in tongue squamous cell carcinoma. Int. J. Cancer 135, 2282-2293 (2014).

92. Zhang, H. et al. Long non-coding RNA HOTTIP is correlated with progression and prognosis in tongue squamous cell carcinoma. Tumour Biol. 36, 8805-8809 (2015).

93. Huang, W. et al. Long non-coding RNA NKILA inhibits migration and invasion of tongue squamous cell carcinoma cells via suppressing epithelial-mesenchymal transition. Oncotarget 7, 62520-62532 (2016).

94. Li, Z. Q., Zou, R., Ouyang, K. X. \& Ai, W. J. An in vitro study of the long non-coding RNA TUG1 in tongue squamous cell carcinoma. J. Oral. Pathol. Med. 46, 956-960 (2017).

95. Ouyang, K. X. et al. TUC338 overexpression leads to enhanced proliferation and reduced apoptosis in tongue squamous cell carcinoma cells in vitro. J. Oral. Maxillofac. Surg. 75, 423-428 (2017).

96. $\mathrm{Yu}, \mathrm{J}$. et al. Upregulated long non-coding RNA LINC00152 expression is associated with progression and poor prognosis of tongue squamous cell carcinoma. J. Cancer 8, 523-530 (2017).

97. $\mathrm{Yu}, \mathrm{J}$. et al. Overexpression long non-coding RNA LINC00673 is associated with poor prognosis and promotes invasion and metastasis in tongue squamous cell carcinoma. Oncotarget 8, 16621-16632 (2017).

98. Fang, Z. et al. Long non-coding RNA MALAT-1 modulates metastatic potential of tongue squamous cell carcinomas partially through the regulation of small proline rich proteins. BMC Cancer 16, 706 (2016).

99. Zhang, T. H. et al. Long non-coding RNA MALAT1 interacts with miR-124 and modulates tongue cancer growth by targeting JAG1. Oncol. Rep. 37, 2087-2094 (2017).

100. Hartig, G. et al. Fluorescent in situ hybridizaton evaluation of $\mathrm{p} 53$ gene deletions at a tumor interface of lingual carcinoma. Laryngoscope 110, 1474-1478 (2000).

101. Fujii, M., Ishiguro, R., Yamashita, T. \& Tashiro, M. Cyclin D1 amplification correlates with early recurrence of squamous cell carcinoma of the tongue. Cancer Lett. 172, 187-192 (2001).

102. Goto, H., Kawano, K., Kobayashi, I., Sakai, H. \& Yanagisawa, S. Expression of cyclin D1 and GSK-3beta and their predictive value of prognosis in squamous cell carcinomas of the tongue. Oral. Oncol. 38, 549-556 (2002).

103. Hannen, E. J. et al. Different chromosomal imbalances in metastasized and nonmetastasized tongue carcinomas identified by comparative genomic hybridization. Oral. Oncol. 40, 364-371 (2004).

104. Shimizu, Y., Kondo, S., Shirai, A., Furukawa, M. \& Yoshizaki, T. A single nucleotide polymorphism in the matrix metalloproteinase- 1 and interleukin-8 gene promoter predicts poor prognosis in tongue cancer. Auris Nasus Larynx 35, 381-389 (2008).
105. Angiero, F. et al. Comparative analysis of c-erbB-2 (HER-2/neu) in squamous cell carcinoma of the tongue: does over-expression exist? And what is its correlation with traditional diagnostic parameters? J. Oral. Pathol. Med. 37, 145-150 (2008).

106. Ryott, M. et al. EGFR protein overexpression and gene copy number increases in oral tongue squamous cell carcinoma. Eur. J. Cancer 45, 1700-1708 (2009).

107. Prapinjumrune, $C$. et al. DNA amplification and expression of FADD in oral squamous cell carcinoma. J. Oral. Pathol. Med. 39, 525-532 (2010).

108. Aida, J. et al. Telomere lengths in the oral epithelia with and without carcinoma. Eur. J. Cancer 46, 430-438 (2010).

109. Supic, G., Kozomara, R., Jovic, N., Zeljic, K. \& Magic, Z. Hypermethylation of RUNX3 but not WIF1 gene and its association with stage and nodal status of tongue cancers. Oral. Dis. 17, 794-800 (2011).

110. Young, R. J. et al. Frequency of fibroblast growth factor receptor 1 gene amplification in oral tongue squamous cell carcinomas and associations with clinical features and patient outcome. Oral. Oncol. 49, 576-581 (2013).

111. Mostaan, L. V. et al. Survivin gene polymorphism association with tongue squamous cell carcinoma. Genet. Test. Mol. Biomark. 17, 74-77 (2013).

112. Adduri, R. S. et al. TP53 Pro72 allele is enriched in oral tongue cancer and frequently mutated in esophageal cancer in India. PLOS ONE 9, e114002 (2014).

113. Tan, D. S. et al. Tongue carcinoma infrequently harbor common actionable genetic alterations. BMC Cancer 14, 679 (2014).

114. Lim, A. M. et al. Differential mechanisms of CDKN2A (p16) alteration in oral tongue squamous cell carcinomas and correlation with patient outcome. Int. J. Cancer 135, 887-895 (2014).

115. Morita, T. et al. Characterizing genetic transitions of copy number alterations and allelic imbalances in oral tongue carcinoma metastasis. Genes Chromosomes Cancer 55, 975-986 (2016).

116. Kakuya, T. et al. Prognostic significance of gene amplification of ACTN4 in stage I and II oral tongue cancer. Int. J. Oral. Maxillofac. Surg. 46, 968-976 (2017).

117. Atula, S., Kurvinen, K., Grenman, R. \& Syrjanen, S. SSCP pattern indicative for p53 mutation is related to advanced stage and high-grade of tongue cancer. Eur. J. Cancer B. Oral. Oncol. 32B, 222-229 (1996).

118. Heaton, C. M., Durr, M. L., Tetsu, O., van Zante, A. \& Wang, S. J. TP53 and CDKN2a mutations in never-smoker oral tongue squamous cell carcinoma. Laryngoscope 124, E267-E273 (2014).

119. Adduri, R. et al. P53 nuclear stabilization is associated with FHIT loss and younger age of onset in squamous cell carcinoma of oral tongue. BMC Clin. Pathol. 14, 37 (2014).

120. Krishnan, N. et al. Integrated analysis of oral tongue squamous cell carcinoma identifies key variants and pathways linked to risk habits, HPV, clinical parameters and tumor recurrence. F1000Res. 4, 1215 (2015).

121. Vettore, A. L. et al. Mutational landscapes of tongue carcinoma reveal recurrent mutations in genes of therapeutic and prognostic relevance. Genome Med. 7, 98 (2015). 